\title{
Neue pathogenetische Konzepte und pharmakolo- gische Studien zur adjuvanten Therapie bei schwerer Pneumonie
}

\author{
New Pathogenetic Concepts and Pharmacological Studies on Adjuvant Therapy \\ in Severe Pneumonia
}

Autoren

Institut
J. Lienau, H. Müller-Redetzky, N. Suttorp, M. Witzenrath

Charité - Universitätsmedizin Berlin, Medizinische Klinik mit Schwerpunkt Infektiologie und Pneumologie eingereicht $\quad 2.10 .2015$ akzeptiert nach Revision 13.10.2015

Bibliografie

DOI http://dx.doi.org/

10.1055/s-0035-1563788

Online-Publikation: 4.12.2015

Pneumologie 2016; 70: 372-378

(c) Georg Thieme Verlag KG

Stuttgart · New York

ISSN 0934-8387

Korrespondenzadresse

Prof. Dr. med.

Martin Witzenrath

Charité - Universitätsmedizin

Berlin, Medizinische Klinik mit

Schwerpunkt Infektiologie und

Pneumologie

Charitéplatz 1

10117 Berlin

Martin.Witzenrath@charite.de

Serienherausgeber

M. Witzenrath, Berlin

\section{Zusammenfassung}

$\nabla$

Das akute Lungenversagen bei schwerer Pneumonie ist Folge einer Aktivierung des angeborenen Immunsystems mit schädigender Hyperinflammation, Verlust der alveolokapillären Barrierefunktion sowie mikrozirkulatorischen Störungen. Bislang existiert neben der antimikrobiellen Therapie keine pharmakologische Strategie zur Prävention oder Behandlung des akuten Lungenversagens bei Pneumonie. In präklinischen Studien konnten vielversprechende Zielmoleküle identifiziert werden, und neuartige therapeutische Ansätze zeigten bei Pneumonie protektive Effekte hinsichtlich der Entwicklung eines akuten Lungenschadens. In diesem Übersichtsartikel werden einige adjuvante therapeutische Strategien bei Pneumonie vorgestellt und Zukunftsperspektiven diskutiert.

\section{Einleitung}

Die Lungenentzündung ist weltweit die häufigste zum Tode führende Infektionskrankheit und verursacht $15 \%$ aller Todesfälle bei Kindern unter 5 Jahren [1]. Auch in Deutschland zählt die Pneumonie zu den bedeutenden Volkskrankheiten mit jährlich ca. 600000 ambulant und ca. 100000 stationär erworbenen Erkrankungen [2]. Ungefähr ein Drittel aller Patienten mit ambulant erworbener Pneumonie wird in Deutschland stationär behandelt, mit einer seit Einführung der antimikrobiellen Therapie weitgehend unverändert hohen Letalität von ca. 13-14\% [2]. Patienten mit Pneumonie können akutes Lungenversagen, Sepsis mit Multiorganversagen oder kardiovaskuläre Komplikationen entwickeln. Diese Komplikationen treten auch unter antimikrobieller Therapie auf, auch wenn diese nachweislich gegen das für die Pneumonie ursächliche Pathogen wirksam ist, und führen häufig kurz- oder mittelfristig zum Tod. Somit stellt die Behandlung der Pneumonie

\section{Abstract \\ $\nabla$}

Acute lung injury secondary to pneumonia results from inadequate activation of the innate immune system with hyperinflammation and alveolarcapillary barrier dysfunction. To date, effective strategies for prevention or treatment of acute lung injury in pneumonia besides antibiotics are lacking. In preclinical studies, promising therapeutic targets have been identified and novel strategies demonstrated to protect against lung failure in pneumonia. This review highlights some adjuvant therapeutic strategies for modulation of inflammation and stabilization of lung barrier function in pneumonia.

weiterhin eine besondere medizinische und sozioökonomische Herausforderung dar, die durch die steigende Rate multiresistenter bakterieller Pneumonie-Erreger und das Auftreten viraler Erreger mit pandemischem Potenzial erschwert wird.

Das angeborene Immunsystem ist verantwortlich für die sofortige Entzündungs- und Abwehrreaktion auf Infektionen und Gewebeschäden. Sogenannte Mustererkennungsrezeptoren (pattern recognition receptors, PRRs) erkennen konservierte mikrobielle Moleküle (pathogen-associated molecular patterns, PAMPs) sowie endogene Moleküle, die bei Gewebeschäden freigesetzt werden (danger-associated molecular patterns, DAMPs) $[3,4]$. Die Aktivierung der PRRs induziert die Synthese proinflammatorischer Zytokine, Interferone und Chemokine [5], die wiederum ortsständige Zellen aktivieren und Makrophagen und neutrophile Granulozyten rekrutieren, was schließlich zur Elimination der Pathogene und infizierten Zellen führt. Trotz antibiotischer Therapie kann es je- 
doch zu einer Überaktivierung des Immunsystems kommen und zwar durch anhaltende Freisetzung der PAMPs von absterbenden Bakterien bzw. der DAMPs von geschädigten Zellen. Infolge dessen kommt es zur Überproduktion von Entzündungsmediatoren, unkontrollierter Rekrutierung und Aktivierung von Leukozyten, inadäquater Aktivierung von Komplement- und Koagulationskaskaden sowie zur Störung der alveolokapillären Barrierefunktion. Durch erhöhte Permeabilität entwickelt sich ein proteinreiches Lungenödem, und es kommt zum akuten Lungenversagen (acute respiratory distress syndrome, ARDS) (siehe Review [6]), das eine Mortalität von 27 - 45\% aufweist [7].

Bei Patienten mit akuter respiratorischer Insuffizienz ist der Einsatz der maschinellen Beatmung zur Aufrechterhaltung des Gasaustausches eine alternativlose, lebensrettende Maßnahme. Weltweit wird auf Intensivstationen jeder dritte Patient maschinell beatmet [8], wobei die Pneumonie eine der führenden Ursachen darstellt [9-14]. Obwohl die maschinelle Beatmung durch Verbesserung oder Aufrechterhaltung des Gasaustausches und der Ventilation lebensrettend oder lebenserhaltend sein kann, birgt die Anwendung der unphysiologischen Überdruckbeatmung auch Risiken und kann die pulmonale Inflammation und Störung der Barrierefunktion verstärken [15]. Die Reduktion des Tidalvolumens („Lungenprotektive“ Beatmung) ist bis heute die einzige Intervention, welche die Mortalität bei ARDS nachweislich senkt [16]. Auch die Applikation eines adäquaten positiven endexspiratorischen Drucks (PEEP) verbessert den Behandlungserfolg bei ARDS-Patienten, wobei das optimale PEEP-Niveau noch unklar ist $[17,18]$. Darüber hinaus können eine frühzeitige Bauchlagerung sowie die Gabe von Muskelrelaxanzien zur Reduktion der Mortalität bei Patienten mit schwerem ARDS beitragen [19-22].

Die präklinische Forschung bemüht sich erfolgreich, pathophysiologische Zusammenhänge zu klären, dennoch sind neuartige präventive und therapeutische Möglichkeiten zur Behandlung von Pneumonie und akutem Lungenversagen zusätzlich zu Antibiotika bisher klinisch nicht etabliert. Der vorliegende kurze Übersichtsartikel fasst einige ausgewählte präklinische und frühe klinische Daten aus Studien zur Modulation der Immunantwort und Therapie der pulmonalen Barrierestörung bei Pneumonie zusammen.

\section{Immunmodulatorische Therapie}

\section{$\nabla$}

\section{Kortikosteroide}

In den letzten Jahrzehnten standen Kortikosteroide zur Behandlung der Pneumonie immer wieder im Fokus klinischer Forschung, mit unterschiedlichen Ergebnissen. In einer 2012 veröffentlichten Metaanalyse von neun RCTs aus den Jahren 1952 bis 2011 mit insgesamt 1001 Patienten zeigte sich eine signifikante Reduktion der Mortalität nach adjuvanter Kortikosteroid-Therapie von Patienten mit schwerer CAP [23]. Dieses Ergebnis basierte jedoch primär auf einer Single-Center-Studie mit nur 80 Patienten [24], wohingegen sich in den anderen Studien keine deutliche Verbesserung der Mortalität zeigte. In der 2007 veröffentlichten retrospektiven Analyse von Garcia-Vidal und Kollegen zeigte sich ebenfalls eine reduzierte Mortalität in Patienten mit schwerer CAP nach adjuvanter Kortikosteroid-Therapie [25]. Auch die Ergebnisse aus einer japanischen Datenbank von beatmeten CAP-Patienten zeigen eine reduzierte Mortalität in einer Untergruppe von CAP-Patienten mit Schock, gekennzeichnet durch Katecholamin-Gabe [26]. Die Interpretation dieser Daten ist je- doch schwierig, da zum einen unterschiedliche KortikosteroidDosen verwendet wurden und zum anderen Informationen über zahlreiche wichtige Parameter fehlen, einschließlich Laktat-Spiegel, Blutdruck, Flüssigkeitssubstitution sowie antibiotische Versorgung.

Kürzlich wurde eine multizentrische randomisierte Studie aus der Schweiz publiziert. Im Zeitraum 2009-2014 wurden 785 Patienten mit Prednisolon (50 mg täglich für 7 Tage) oder Placebo (1:1) adjuvant behandelt [27]. Die Behandlung mit Prednisolon führte zu einer schnelleren klinischen Stabilität und einem kürzeren Krankenhausaufenthalt, war jedoch mit häufigerer insulinpflichtiger Hyperglykämie assoziiert. Es liegt allerdings die Vermutung nahe, dass Krankheitssymptome durch Prednisolon maskiert und infolge dessen Patienten früher entlassen wurden. Ebenfalls 2015 wurde eine randomisierte kontrollierte Studie von Torres und Kollegen publiziert, die in drei spanischen Zentren Patienten mit „proinflammatorischem Phänotyp“ der CAP einschloss, definiert durch CRP-Spiegel größer als $150 \mathrm{mg} / \mathrm{L}$ [28]. In dieser Studie waren 61 Patienten eingeschlossen, die mit 0,5 mg Methylprednisolon pro kg Körpergewicht zweimal täglich an fünf aufeinanderfolgenden Tagen behandelt wurden, sowie 59 Patienten der Placebogruppe. Es zeigte sich eine signifikante Reduktion des späten Therapieversagens, hauptsächlich basierend auf einer Reduktion radiologischer Zeichen der Pneumonie im Zeitraum 72 Stunden bis 7 Tage nach Einschluss. Dieselbe Arbeitsgruppe hatte zuvor gezeigt, dass Therapieversagen mit Mortalität [29], dass CRP Level über $150 \mathrm{mg} / \mathrm{L}$ mit Therapieversagen [30] und dass radiologische Progression sowohl mit Therapieversagen als auch mit Mortalität assoziiert ist [29,31]. Dennoch konnte in der aktuellen Studie kein Einfluss der Methylprednisolontherapie auf den klinischen Verlauf innerhalb der ersten 72 Stunden, die Beatmungsnotwendigkeit oder die KrankenhausMortalität beobachtet werden. Auch ist unklar, ob Methylprednisolon die Entstehung des akuten Lungenversagens reduzierte, eine weniger schädliche lokale physiologische Inflammationsreaktion behinderte oder die Infiltrat-Auflösung unterstützte. Bemerkenswert ist, dass die Inzidenz hyperglykämischer Entgleisungen in der Methylprednisolon-Gruppe nicht signifikant anstieg.

Zusammenfassend könnten Kortikosteroide in der Behandlung von Pneumoniepatienten mit schwerer pulmonaler oder systemischer Inflammation einen Vorteil bieten. Es ist naheliegend, dass dies nur für eine kleine Gruppe von Pneumoniepatienten gilt, die im Sinne eines „personalisierten“ Ansatzes zunächst identifiziert und für die der vermutete Vorteil in größeren Studien nachgewiesen werden muss, bevor eine eindeutige Empfehlung ausgesprochen werden kann.

\section{Signaltransduktion von Pattern Recognition Receptors} (Mustererkennungsrezeptoren; PRRs)

Der Fokus zahlreicher Studien lag und liegt noch immer auf der Entwicklung von Strategien, welche die PRR-Signaltransduktion modulieren. Große Hoffnungen wurden in die Entwicklung von Eritoran, einem synthetischen Inhibitor der Toll-like Rezeptor 4 (TLR4)-Signaltransduktion gesetzt, da verschiedene experimentelle Studien demonstrierten, dass die Aktivierung von TLR4 vaskuläre Permeabilität auslöst [32-36]. In experimentellen Tiermodellen [37] und auch in gesunden Probanden nach einer LPSBolusinfusion [38] zeigte sich eine Reduktion der pulmonalen Inflammation nach Eritoran-Gabe. Vielversprechende Ergebnisse zeigte auch eine klinische Studie der Phase II an Patienten mit schwerer Sepsis, die nach Eritoran-Gabe einen Trend zu einer ge- 
ringeren Mortalität aufwiesen [39]. Jedoch waren die Ergebnisse in einer multinationalen Studie der Phase III an Patienten mit schwerer Sepsis enttäuschend. Hier zeigte Eritoran keinen bedeutenden Effekt auf die 28-Tage-Mortalität oder relevante sekundäre Outcome-Parameter [40], was den therapeutischen Einsatz von TLR4-Antagonisten im Rahmen von Sepsis und Organversagen einschließlich ARDS grundsätzlich in Frage stellt. Betrachtet man die molekularen Zusammenhänge der PRR-Signaltransduktion, so sind diese Ergebnisse möglicherweise dadurch erklärbar, dass eine Vielzahl von oftmals gleichzeitig auftretenden PAMPs und DAMPS Liganden für unterschiedliche PRRs darstellen, deren Aktivierung schließlich zur NF-kB-vermittelten Transkription inflammatorischer Gene führt. Aus diesem Grund erscheint es sinnvoller, weiter downstream in den Inflammationskaskaden zentrale Effektoren zu modulieren, anstatt auf einen einzelnen PRR zu fokussieren [40].

\section{GM-CSF}

Ein weiterer Ansatz, die Immunantwort zu modulieren, ist die Behandlung mit dem hämatopoetischen Wachstumsfaktor GMCSF (granulocyte-macrophage colony-stimulating factor). Dieser besitzt immunregulatorische Eigenschaften, abhängig von der Konzentration und Anwesenheit anderer Zytokine, sowie dem Kontext der Immunantwort (siehe Review [41]). Gebildet wird GM-CSF von verschiedenen Zelltypen einschließlich Epithelzellen und Makrophagen. In einer prospektiven, randomisierten, multizentrischen Studie an Sepsis-Patienten zeigte sich ein positiver Effekt der GM-CSF Therapie auf die Sepsis-assoziierte Immunsuppression sowie den klinischen Verlauf [42]. In verschiedenen experimentellen Infektionsmodellen einschließlich Influenzavirus, Klebsiella pneumoniae und Streptococcus pneumoniae verbesserte GM-CSF die pulmonale Immunabwehr und zeigte einen protektiven Effekt hinsichtlich der Entwicklung eines Lungenschadens [43-46].

Die Ergebnisse klinischer Studien an Patienten mit Lungenversagen sind jedoch uneinheitlich. In zwei randomisierten klinischen Studien der Phase II hatten Patienten mit Sepsis-induziertem ARDS keinen Benefit nach systemischer GM-CSF Behandlung [47, 48]. Im Gegensatz dazu zeigen kürzlich veröffentlichte Daten einer unkontrollierten klinischen Pilot-Studie, dass die inhalative GM-CSF Behandlung von Patienten mit Pneumonie-induziertem ARDS zur Verbesserung der pulmonalen Abwehr, der Oxygenierung und des klinischen Verlaufs führte [49]. Weitere Studien sind notwendig und bereits in Planung, um die Eignung von GM-CSF als vielversprechende immunmodulatorische Substanz zur inhalativen Behandlung des Lungenschadens bei Pneumonie zu evaluieren.

\section{Komplement-Inhibitoren}

Das Komplementsystem spielt als Teil des angeborenen Immunsystems eine wichtige Rolle bei der Abwehr von Pathogenen und inflammatorischen Reaktionen [50]. Eine inadäquate Aktivierung des Komplementsystems kann Hyperinflammation und endotheliale Barrieredysfunktion auslösen. Eine wesentliche Rolle hierbei spielt das Komplementaktivierungsprodukt C5a [5153]. Die Inhibition von C5a erwies sich experimentell als vielversprechender Ansatz zur Abschwächung der Immunreaktion des Wirtes. So führte die Neutralisation von C5a in Mausmodellen des akuten Lungenschadens sowie systemischer Entzündungsreaktion zur Reduktion der vaskulären Permeabilität in der Lunge sowie in anderen Organen [54]. Auch bei pneumogener Sepsis mit und ohne maschinelle Beatmung zeigte die Neutralisierung von C5a mittels Spiegelmer einen protektiven Effekt auf Lungenund extrapulmonales Organversagen [55].

\section{Immunmodulatorische Effekte von Antibiotika}

Makroliden und Fluorchinolonen werden immunmodulatorische Eigenschaften zugeschrieben. Die immunmodulatorischen Effekte von Makroliden sind in vitro vielfach untersucht. So können Makrolide die inflammatorischen Reaktionen gegenüber einer Vielzahl Stimuli abschwächen, die Bildung bestimmter Virulenzfaktoren (z.B. Pneumolysin) reduzieren [56] und in die mikrobielle Kommunikation (quorum sensing) eingreifen [57].

Die Kombinationstherapie in Form einer Beta-Lactam-MakrolidKombination wird in deutschen und europäischen Leitlinien für sCAP Patienten sowie in den ATS Leitlinien für hospitalisierte CAP-Patienten empfohlen [58-60]. Diese Empfehlung basiert auf (1) einem potentiell synergistischen bakteriziden Effekt, (2) der Erfassung atypischer Pathogene (hauptsächlich Legionella ssp.) und (3) einer möglichen immunmodulatorischen Wirkung. Jedoch stützt sich die Empfehlung hauptsächlich auf retrospektive Analysen, in welchen vorrangig sCAP Patienten einen verbesserten Behandlungserfolg unter Kombinationstherapie zeigten $[61,62]$. Erstaunlicherweise erfolgte in keiner der Studien eine Charakterisierung des inflammatorischen Profils der Patienten.

In einer kürzlich veröffentlichten Metaanalyse wurde ein verbessertes Überleben nach Beta-Lactam-Makrolid-Kombinationstherapie beschrieben, jedoch nur im Vergleich zur Beta-LactamMonotherapie, nicht aber im Vergleich zur Therapie mit einem Fluorchinolon oder wenn die Analysen auf die wenigen inkludierten RCT-Studien beschränkt wurden. Darüber hinaus waren schwerkranke Intensivpatienten ausgeschlossen [63].

In einer randomisierten kontrollierten Nichtunterlegenheitsstudie aus dem Jahr 2014 an hospitalisierten Patienten mit CAP (54\% CURB > 2, PSI-Klasse V wurde ausgeschlossen) wurde die Beta-Lactam-Monotherapie mit der Beta-Lactam-MakrolidKombinationstherapie verglichen. Dabei wurde der primäre Endpunkt, die Zeit bis zur klinischen Stabilisierung an Tag 7, seltener unter Monotherapie erreicht. Auch hier profitierten hauptsächlich schwerkranke Patienten mit einem CURB-Score über 2 oder der PSI-Klasse IV von der Kombinationstherapie. Erstaunlicherweise traf das auch für Patienten zu, die mit atypischen Pathogenen infiziert waren, obwohl Makrolide auch in der Monotherapiegruppe bei positivem Legionella-Antigen-Nachweis im Urin verabreicht wurden [64].

In einer zweiten im Jahr 2014 veröffentlichten randomisierten kontrollierten Nichtunterlegenheitsstudie an hospitalisierten Patienten mit CAP ohne Indikation zur Intensivtherapie (vergleichbar mit der zuvor genannten Studie) wurde die Beta-Lactam-Monotherapie mit der Beta-Lactam-Makrolid-Kombinationstherapie sowie mit der Fluorchinolon-Monotherapie verglichen. Bezüglich des primären Endpunktes, der 90-Tage-Mortalität, war die Beta-Lactam-Monotherapie der Kombinationstherapie oder Monotherapie mit einem Fluorchinolon nicht unterlegen [65]. Zusammenfassend betrachtet ist ein immunmodulatorischer Effekt von Makroliden bei der Behandlung der unkomplizierten CAP möglich, er scheint aber eine untergeordnete Rolle zu spielen, und eindeutige klinische Evidenz zu erlangen ist schwierig. Leider fehlen RCTs an Patienten mit schwerer CAP. Darüber hinaus wäre es sinnvoll, den inflammatorischen Phänotyp der Patienten zu bestimmen, um zu überprüfen, ob sich die experimentellen Beobachtungen bezüglich der Immunmodulation durch Makrolide auch auf den Menschen übertragen lassen. 
Fluorchinolone mit einem Cyclopropylrest in der N1-Position (z.B. Ciprofloxacin, Moxiflocacin) zeigen in vitro anti-inflammatorische Effekte [66-69]. In eigenen experimentellen Untersuchungen an humanem Lungengewebe und in Mäusen in vivo konnten wir jedoch nach Pneumokokkeninfektion für Moxifloxacin diese Effekte nicht bestätigen [70]. Zudem zeigte sich klinisch die Meropenem-Moxifloxacin-Kombinationstherapie im Vergleich zur Meropenem-Monotherapie in Patienten mit Sepsis (hauptsächlich pneumogene Sepsis) als nicht überlegen [71]. Ein immunmodulatorischer Effekt von Fluorchinolonen in der Pneumonie ist daher derzeit nicht belegt.

\section{Therapie der pulmonalen Barrierestörung}

\section{$\nabla$}

\section{Adrenomedullin}

Adrenomedullin (AM) ist ein körpereigenes Peptid, das als Teil eines Vorläuferproteins (Präpro-AM) von verschiedenen Zelltypen (z.B. Epithelzellen, Endothelzellen, glatte Muskelzellen, Leukozyten) synthetisiert wird. Mäuse, die defizient für AM, den AM-Rezeptor CRLR (Calcitonin Receptor-Like Receptor) oder andere Komponenten des AM-Signalweges sind, sterben frühzeitig an der Entwicklung eines Hydrops fetalis [72 - 75]. Experimentell konnte gezeigt werden, dass die AM-Expression unter inflammatorischen Bedingungen, z. B. bei Sepsis oder akutem Lungenversagen erhöht ist [76-78]. Heterozygot AM-defiziente Mäuse zeigten nach LPS-Behandlung eine verstärkte inflammatorische Antwort sowie ausgeprägtere Organschäden [79]. Umgekehrt führte eine Behandlung mit exogenem AM in eigenen Untersuchungen der Autoren zu einer Stabilisierung der pulmonalvaskulären Barrierefunktion in verschiedenen experimentellen Modellen und zeigte sich protektiv gegenüber der Entwicklung eines beatmungsassoziierten Lungenschadens (ventilator-induced lung injury, VILI) in Mäusen mit oder ohne vorbestehender Pneumonie [80-84].

AM hat somit in verschiedenen komplexen experimentellen Modellen eindrucksvolle Eigenschaften demonstriert und stellt einen äußerst vielversprechenden Kandidaten für eine adjuvante Therapie der Pneumonie dar. Die klinische Evaluation ist in Planung.

\section{Angiopoietine}

Angiopoietin 1 (Ang-1) und Ang-2 spielen eine wichtige Rolle in der Regulation von Angiogenese, Inflammation und Gefäßpermeabilität (siehe Reviews $[85,86]$ ). Ang-1 wird konstitutiv exprimiert und bindet an den endothelial-exprimierten Tyrosinkinaserezeptor Tie2, was zur Stabilisierung interzellulärer Kontakte und Zell-Matrix-Kontakte sowie zur Rekrutierung peri-endothelialer mesenchymaler Zellen führt [87]. Ang-1 fungiert damit als konstitutiver Agonist von Tie2 und ist für den Erhalt der vaskulären Integrität notwendig. Ang-2, ebenfalls Ligand von Tie2, agiert hingegen als funktioneller Antagonist und führt zur Destabilisierung der endothelialen Schranke, Inflammation und Hyperpermeabilität $[88,89]$.

In verschiedenen Studien wurde Ang-2 eine Rolle als potenzieller Marker für die Schwere der Erkrankung zugeschrieben. So konnten Parikh und Kollegen 2006 bereits zeigen, dass die Ang-2Spiegel im Blut bei septischen Patienten im Vergleich zu gesunden Kontrollpersonen erhöht sind [90]. Zudem wurde eine Korrelation zwischen dem Ang-2-Gehalt und der Mortalität beim nicht-infektiös bedingten, nicht aber beim infektiös bedingten akuten Lungenschaden demonstriert [91]. Auch in der Pathoge- nese der Sepsis scheint Ang-2 eine Rolle zu spielen. So konnten David und Kollegen zeigen, dass heterozygote Mäuse mit nur einem funktionsfähigen Ang-2 Allel geringere Lungen- und Organschäden sowie eine geringere Mortalität in verschiedenen Sepsis-Modellen aufwiesen [92]. Analog dazu führte die Behandlung von Mäusen mit Antikörpern gegen Ang-2 zur Reduktion von Permeabilität und Mortalität nach LPS-Injektion [93]. Im Gegensatz zu Ang-2 zeigte Ang-1 in zahlreichen präklinischen Studien einen protektiven anti-inflammatorischen Effekt. Die Behandlung mit Ang-1 oder transgene Überexpression von Ang-1 führte in experimentellen Modellen des akuten Lungenschadens zu einer Reduktion der Expression von Zytokinen und Adhäsionsmolekülen, PMN-Infiltration und Permeabilität in der Lunge [94 -97]. Darüber hinaus wurden in zahlreichen Studien die Barriere-stabilisierenden Effekte von Ang-1 eindrucksvoll demonstriert [94-96, $98-100]$.

Vielversprechend ist die Entwicklung von Vasculotide, einem kurzen synthetischen Tie2 Rezeptor-Agonisten, der die Bindung von Ang-1 und Ang-2 inhibiert [101]. Das therapeutische Potenzial von Vasculotide konnte bereits in murinen Modellen der Endotoxämie, der abdominalen Sepsis und jüngst auch der Influenza-Pneumonie demonstriert werden. Hier zeigte sich nach Applikation von Vasculotide eine verbesserte Barrierefunktion sowie reduzierte Mortalität [102-104].

\section{Stammzellbasierte Therapie}

Stammzellen können durch ihr Potenzial zur Selbsterneuerung und Differenzierung in eine Vielzahl unterschiedlicher Zelltypen zur Regeneration geschädigter Gewebe und Organe beitragen [105]. Im Modell des LPS-induzierten Lungenschadens konnte gezeigt werden, dass sich Stammzellen nach Transplantation ins Lungengewebe integrieren, mit Nachbarzellen interagieren und so die endotheliale und epitheliale Regeneration unterstützen. Es ist anzunehmen, dass die transplantierten Zellen dabei von Chemokinen im geschädigten Gewebe angelockt wurden, da im gesunden Lungengewebe exogene Stammzellen nicht detektierbar waren [106]. Neben der direkten Zellintegration können Stammzellen auch die Immunantwort modulieren. Bemerkenswerterweise werden überschießende inflammatorische Reaktionen reduziert, während gleichzeitig die Fähigkeit des Wirtes, Pathogene zu bekämpfen, aufrechterhalten wird [105]. Für die beschriebenen positiven Effekte scheinen hauptsächlich parakrine Mediatoren der Stammzellen sowie von Stammzellen sezernierte Mitochondrien-enthaltende Mikrovesikel verantwortlich zu sein $[107,108]$.

Eine Untergruppe hämatopoetischer Stammzellen bilden endotheliale Progenitorzellen (endothelial progenitor cells, EPCs), die ausschließlich in Endothelzellen differenzieren können. EPCs können aus verschiedenen Quellen isoliert werden, z.B. aus zirkulierenden mononukleären Zellen, Knochenmark oder Nabelschnurblut. In ARDS-Patienten konnte eine erhöhte Anzahl zirkulierender EPCs nachgewiesen werden, die mit einem verbesserten Überleben der Patienten assoziiert war [109]. Jedoch zeigte sich eine eingeschränkte Funktionalität (reduzierte Proliferation, Adhäsion, Migration, angiogene Eigenschaft) endogener EPCs in septischen Individuen $[110,111]$.

Um die positiven Effekte der EPCs zu erhalten, wurden experimentell zwei Strategien verfolgt. Die erste Strategie basiert auf der Transplantation von EPCs, die aus gesunden Spendern isoliert wurden. Die EPC-Transplantation in Tiermodellen des akuten Lungenversagens führte, wahrscheinlich durch Reendothelialisierung des geschädigten pulmonalen Gefäßsystems, zur Reduk- 
tion der Bildung von Ödem und hyalinen Membranen [106,112]. Zur Verbesserung der Funktionalität der EPCs wurde im zweiten Ansatz die autologe EPC-Transplantation mit der Applikation des Chemokins SDF-1 $\alpha$ kombiniert [113]. Im murinen Modell der polymikrobiellen Sepsis führte die Kombination von exogenen EPCs und SDF- $1 \alpha$ zur Verbesserung der pulmonalen Gefäßintegrität und des Überlebens [113]. Die Transplantation von EPCs als präventive oder therapeutische Strategie bei ARDS erscheint sehr vielversprechend, weitere präklinische Untersuchungen von Effektivität und Wirkungsmechanismus sind jedoch notwendig. Derzeit werden humane, allogene mesenchymale Stammzellen aus dem Knochenmark als adjuvante Therapie zur Behandlung von ARDS in einer klinischen Studie der Phase I (NCT01775774) sowie in einer multizentrischen Phase-II-Studie (NCT02097641) analysiert.

\section{Ausblick}

Angesichts der seit Jahrzehnten unveränderten, inakzeptabel hohen Letalität der Pneumonie ist die Identifikation zentraler Pathogenitätsmechanismen und die erfolgreiche Translation neuer Therapiestrategien in die Klinik von besonderer Bedeutung. Die pathophysiologische Relevanz von Hyperinflammation und Barrieredysfunktion bei Pneumonie-assoziiertem ARDS ist lange bekannt, und neuartige Strategien, welche die pathophysiologischen Mechanismen modulieren, wurden bereits erfolgreich in präklinischen proof-of-concept Modellen getestet. Allerdings war keine dieser Strategien bisher klinisch erfolgreich. Um die Effizienz der Translation von präklinischen Forschungsergebnissen in die Klinik zu erhöhen, sollten einige Aspekte beachtet werden [114]. Erstens sollten zur adäquaten Abbildung des Pneumoniepatienten verschiedene präklinische Modelle von hinreichender Komplexität kombiniert werden, die an die jeweilige klinische Situation angepasst sind. Zweitens sollte man sich vor Augen führen, dass die rasche Wiederherstellung der endothelialen Barrierefunktion im Stadium der fortgeschrittenen Endothelschädigung ein fast utopisches Ziel darstellt. Aus diesem Grund sollten Pneumoniepatienten möglichst bereits bei der Diagnosestellung in Risikogruppen für eine ARDS-Entwicklung eingeteilt werden, und die Behandlung sollte dann nötigenfalls ohne zeitliche Verzögerung „präventiv“ erfolgen. Und drittens sollten Daten von klinischen Studien, patientenbezogenen Omics-Analysen, präklinischen Studien u. a. in einem systemmedizinischen Ansatz integriert werden, um mathematische Modelle zu generieren. Diese Modelle können unser Verständnis der Krankheitsvorgänge verbessern, neue therapeutische Ansätze identifizieren und Patientenpopulationen erkennen, die von spezifischen Strategien profitieren können. Mittelfristig können sie zudem zur individuellen Risikostratifizierung beitragen. CAPSyS, ein durch das Bundesministerium für Bildung und Forschung gefördertes Netzwerk mit dem Fokus auf die „Systemmedizin der Pneumonie“, hat die Arbeit mit eben diesem Ziel im Jahr 2014 aufgenommen.

\section{Interessenkonflikt}

$\nabla$

Die Autoren geben an, dass kein Interessenkonflikt besteht.

\section{Danksagung}

Diese Übersichtsarbeit wurde finanziell durch das Netzwerk CAPSyS (Systemmedizin der Pneumonie; e:Med; Bundesministerium für Bildung und Forschung) unterstützt. Sie basiert in Teilen auf einer früheren, englischsprachigen Übersichtsarbeit der Autoren [114].

\section{Literatur}

1 WHO. Pneumonia. Fact sheet $\mathrm{N}^{\circ} 331$. 2015: http://www.who.int/mediacentre/factsheets/fs331/en/ [Date last updated: November 2014. Date last accessed: March 30]

2 Hoffken G, Lorenz J, Kern $W$ et al. [Epidemiology, diagnosis, antimicrobial therapy and management of community-acquired pneumonia and lower respiratory tract infections in adults. Guidelines of the Paul-Ehrlich-Society for Chemotherapy, the German Respiratory Society, the German Society for Infectiology and the Competence Network CAPNETZ Germany]. Pneumologie 2009; 63: e1 -68

3 Kawai T, Akira S. Toll-like receptors and their crosstalk with other innate receptors in infection and immunity. Immunity 2011; 34: $637-$ 650

4 Rock KL, Lai JJ, Kono $\mathrm{H}$. Innate and adaptive immune responses to cell death. Immunol Rev 2011; 243: 191 - 205

5 Opitz B, van Laak V, Eitel J et al. Innate immune recognition in infectious and noninfectious diseases of the lung. Am J Respir Crit Care Med 2010; 181: 1294-1309

6 Muller-Redetzky HC, Suttorp N, Witzenrath M. Dynamics of pulmonary endothelial barrier function in acute inflammation: mechanisms and therapeutic perspectives. Cell Tissue Res 2014; 355: 657-673

7 Ranieri VM, Rubenfeld GD, Thompson BT et al. Acute respiratory distress syndrome: the Berlin Definition. JAMA 2012; 307: 2526-2533

8 Esteban A, Anzueto A, Frutos $F$ et al. Characteristics and outcomes in adult patients receiving mechanical ventilation: a 28-day international study. JAMA 2002; 287: 345-355

9 Amato MB, Barbas CS, Medeiros DM et al. Effect of a protective-ventilation strategy on mortality in the acute respiratory distress syndrome. N Engl J Med 1998; 338: 347 - 354

10 Brochard L, Roudot-Thoraval F, Roupie E et al. Tidal volume reduction for prevention of ventilator-induced lung injury in acute respiratory distress syndrome. The Multicenter Trail Group on Tidal Volume reduction in ARDS. Am J Respir Crit Care Med 1998; 158: 1831-1838

11 Brower RG, Shanholtz CB, Fessler HE et al. Prospective, randomized, controlled clinical trial comparing traditional versus reduced tidal volume ventilation in acute respiratory distress syndrome patients. Crit Care Med 1999; 27: 1492 - 1498

12 Brower RG, Lanken PN, MacIntyre $N$ et al. Higher versus lower positive end-expiratory pressures in patients with the acute respiratory distress syndrome. N Engl J Med 2004; 351: 327-336

13 Villar J, Kacmarek RM, Perez-Mendez L et al. A high positive end-expiratory pressure, low tidal volume ventilatory strategy improves outcome in persistent acute respiratory distress syndrome: a randomized, controlled trial. Crit Care Med 2006; 34: 1311-1318

14 Ventilation with lower tidal volumes as compared with traditional tidal volumes for acute lung injury and the acute respiratory distress syndrome. The Acute Respiratory Distress Syndrome Network. N Engl J Med 2000; 342: $1301-1308$

15 Verbrugge SJ, Lachmann B, Kesecioglu J. Lung protective ventilatory strategies in acute lung injury and acute respiratory distress syndrome: from experimental findings to clinical application. Clin Physiol Funct Imaging 2007; 27: 67-90

16 Network TARDS. Ventilation with lower tidal volumes as compared with traditional tidal volumes for acute lung injury and the acute respiratory distress syndrome. The Acute Respiratory Distress Syndrome Network. N Engl J Med 2000; 342: 1301 - 1308

17 Briel M, Meade M, Mercat A et al. Higher vs lower positive end-expiratory pressure in patients with acute lung injury and acute respiratory distress syndrome: systematic review and meta-analysis. JAMA 2010; 303: $865-873$

18 Talmor D, Sarge T, Malhotra A et al. Mechanical ventilation guided by esophageal pressure in acute lung injury. $\mathrm{N}$ Engl J Med 2008; 359: $2095-2104$ 
19 Alhazzani W, Alshahrani M, Jaeschke R et al. Neuromuscular blocking agents in acute respiratory distress syndrome: a systematic review and meta-analysis of randomized controlled trials. Crit Care 2013; 17: R43

20 Guerin C, Reignier J, Richard JC et al. Prone positioning in severe acute respiratory distress syndrome. N Engl J Med 2013; 368: 2159-2168

21 Papazian L, Forel JM, Gacouin A et al. Neuromuscular blockers in early acute respiratory distress syndrome. N Engl J Med 2010; 363: 1107 1116

22 Sud S, Friedrich JO, Taccone P et al. Prone ventilation reduces mortality in patients with acute respiratory failure and severe hypoxemia: systematic review and meta-analysis. Intensive Care Med 2010; 36: 585 599

23 Nie W, Zhang Y, Cheng J et al. Corticosteroids in the treatment of community-acquired pneumonia in adults: a meta-analysis. PloS one 2012; 7: e47926

24 Confalonieri $M$, Urbino $R$, Potena $A$ et al. Hydrocortisone infusion for severe community-acquired pneumonia: a preliminary randomized study. Am J Respir Crit Care Med 2005; 171: 242 - 248

25 Garcia-Vidal C, Calbo E, Pascual V et al. Effects of systemic steroids in patients with severe community-acquired pneumonia. Eur Respir J 2007; 30: 951 - 956

26 Tagami T, Matsui H, Horiguchi $H$ et al. Low-dose corticosteroid use and mortality in severe community-acquired pneumonia patients. Eur Respir J 2015; 45: $463-472$

27 Blum CA, Nigro N, Briel M et al. Adjunct prednisone therapy for patients with community-acquired pneumonia: a multicentre, double-blind, randomised, placebo-controlled trial. Lancet 2015; 385: 1511 - 1518

28 Torres $A$, Sibila 0 , Ferrer $M$ et al. Effect of corticosteroids on treatment failure among hospitalized patients with severe community-acquired pneumonia and high inflammatory response: a randomized clinical trial. JAMA 2015; 313: 677-686

29 Menendez $R$, Torres $A$, Zalacain $R$ et al. Risk factors of treatment failure in community acquired pneumonia: implications for disease outcome. Thorax 2004; 59: 960 - 965

30 Menendez R, Cavalcanti $M$, Reyes $S$ et al. Markers of treatment failure in hospitalised community acquired pneumonia. Thorax 2008; 63: 447 452

31 Lisboa T, Blot S, Waterer GW et al. Radiologic progression of pulmonary infiltrates predicts a worse prognosis in severe community-acquired pneumonia than bacteremia. Chest 2009; 135: 165-172

32 Ben DF, Yu XY, Ji GY et al. TLR4 mediates lung injury and inflammation in intestinal ischemia-reperfusion. J Surg Res 2012; 174: 326-333

33 Hilberath JN, Carlo T, Pfeffer MA et al. Resolution of Toll-like receptor 4mediated acute lung injury is linked to eicosanoids and suppressor of cytokine signaling 3. FASEB J 2011; 25: 1827-1835

34 Imai Y, Kuba K, Neely GG et al. Identification of oxidative stress and Toll-like receptor 4 signaling as a key pathway of acute lung injury. Cell 2008; 133: 235-249

35 Tauseef M, Knezevic N, Chava KR et al. TLR4 activation of TRPC6-dependent calcium signaling mediates endotoxin-induced lung vascular permeability and inflammation. J Exp Med 2012; 209: 1953-1968

36 Zanotti G, Casiraghi M, Abano JB et al. Novel critical role of Toll-like receptor 4 in lung ischemia-reperfusion injury and edema. Am J Physiol Lung Cell Mol Physiol 2009; 297: L52-63

37 Mullarkey M, Rose JR, Bristol J et al. Inhibition of endotoxin response by e5564, a novel Toll-like receptor 4-directed endotoxin antagonist. J Pharmacol Exp Ther 2003; 304: 1093 - 1102

38 Lynn M, Rossignol DP, Wheeler JL et al. Blocking of responses to endotoxin by E5564 in healthy volunteers with experimental endotoxemia. J Infect Dis 2003; 187: 631-639

39 Tidswell M, Tillis W, Larosa SP et al. Phase 2 trial of eritoran tetrasodium (E5564), a toll-like receptor 4 antagonist, in patients with severe sepsis. Crit Care Med 2010; 38: $72-83$

40 Opal SM, Laterre PF, Francois B et al. Effect of eritoran, an antagonist of MD2-TLR4, on mortality in patients with severe sepsis: the ACCESS randomized trial. JAMA 2013; 309: 1154-1162

41 Bhattacharya P, Budnick I, Singh M et al. Dual Role of GM-CSF as a ProInflammatory and a Regulatory Cytokine: Implications for Immune Therapy. J Interferon Cytokine Res 2015: Mar 24 [Epub ahead of print]

42 Meisel C, Schefold JC, Pschowski R et al. Granulocyte-macrophage colony-stimulating factor to reverse sepsis-associated immunosuppression: a double-blind, randomized, placebo-controlled multicenter trial. Am J Respir Crit Care Med 2009; 180: 640 -648
43 Huang FF, Barnes PF, Feng Y et al. GM-CSF in the lung protects against lethal influenza infection. Am J Respir Crit Care Med 2011; 184: 259 - 268

44 Standiford LR, Standiford TJ, Newstead MJ et al. TLR4-dependent GMCSF protects against lung injury in Gram-negative bacterial pneumonia. Am J Physiol Lung Cell Mol Physiol 2012; 302: L447 - 454

45 Steinwede $K$, Tempelhof 0 , Bolte $K$ et al. Local delivery of GM-CSF protects mice from lethal pneumococcal pneumonia. J Immunol 2011; 187: $5346-5356$

46 Subramaniam R, Barnes PF, Fletcher $K$ et al. Protecting against post-influenza bacterial pneumonia by increasing phagocyte recruitment and ROS production. J Infect Dis 2014; 209: 1827-1836

47 Paine R3rd, Standiford TJ, Dechert RE et al. A randomized trial of recombinant human granulocyte-macrophage colony stimulating factor for patients with acute lung injury. Crit Care Med 2012; 40: 90 -97

48 Presneill JJ, Harris T, Stewart AG et al. A randomized phase II trial of granulocyte-macrophage colony-stimulating factor therapy in severe sepsis with respiratory dysfunction. Am J Respir Crit Care Med 2002; 166: $138-143$

49 Herold S, Hoegner K, Vadasz I et al. Inhaled granulocyte/macrophage colony-stimulating factor as treatment of pneumonia-associated acute respiratory distress syndrome. Am J Respir Crit Care Med 2014; 189: $609-611$

50 Mastellos D, Morikis D, Isaacs SN et al. Complement: structure, functions, evolution, and viral molecular mimicry. Immunol Res 2003; 27: $367-386$

51 Peng Q Li K, Sacks SH et al. The role of anaphylatoxins C3a and C5a in regulating innate and adaptive immune responses. Inflamm Allergy Drug Targets 2009; 8: 236-246

52 Guo RF, Ward PA. Role of C5a in inflammatory responses. Annu Rev Immunol 2005; 23: $821-852$

53 Wang $R$, Xiao H, Guo R et al. The role of C5a in acute lung injury induced by highly pathogenic viral infections. Emerg Microbes Infect 2015; 4: e28

54 Liu ZM, Zhu SM, Qin XJ et al. Silencing of C5a receptor gene with siRNA for protection from Gram-negative bacterial lipopolysaccharide-induced vascular permeability. Mol Immunol 2010; 47: 1325-1333

55 Müller-Redetzky HC, Kellermann U, Tschernig T et al. Neutralizing the complement component C5a protects against lung injury and extrapulmonary organ injury in pneumococcal pneumonia induced sepsis. Am J Respir Crit Care Med 2014; 189: A1647

56 Anderson R, Steel HC, Cockeran R et al. Clarithromycin alone and in combination with ceftriaxone inhibits the production of pneumolysin by both macrolide-susceptible and macrolide-resistant strains of Streptococcus pneumoniae. J Antimicrob Chemother 2007; 59: 224-229

57 Kanoh S, Rubin BK. Mechanisms of action and clinical application of macrolides as immunomodulatory medications. Clin Microbiol Rev 2010; 23: $590-615$

58 Hoffken G, Lorenz J, Kern W et al. [Epidemiology, diagnosis, antimicrobial therapy and management of community-acquired pneumonia and lower respiratory tract infections in adults. Guidelines of the Paul-Ehrlich-Society for Chemotherapy, the German Respiratory Society, the German Society for Infectiology and the Competence Network CAPNETZ Germany]. Pneumologie 2009; 63: e1 - 68

59 Mandell LA, Wunderink RG, Anzueto A et al. Infectious Diseases Society of America/American Thoracic Society consensus guidelines on the management of community-acquired pneumonia in adults. Clin Infect Dis 2007; 44 (Suppl. 02): S27 - 72

60 Woodhead M, Blasi F, Ewig $S$ et al. Guidelines for the management of adult lower respiratory tract infections - summary. Clin Microbiol Infect 2011; 17 (Suppl. 06): 1 - 24

61 Martinez JA, Horcajada JP, Almela $M$ et al. Addition of a macrolide to a beta-lactam-based empirical antibiotic regimen is associated with lower in-hospital mortality for patients with bacteremic pneumococcal pneumonia. Clin Infect Dis 2003; 36: 389-395

62 Restrepo MI, Mortensen EM, Waterer GW et al. Impact of macrolide therapy on mortality for patients with severe sepsis due to pneumonia. Eur Respir J 2009; 33: 153 - 159

63 Asadi L, Sligl WI, Eurich DT et al. Macrolide-based regimens and mortality in hospitalized patients with community-acquired pneumonia: a systematic review and meta-analysis. Clin Infect Dis 2012; 55: 371 380

64 Garin N, Genne D, Carballo $S$ et al. beta-Lactam monotherapy vs betalactam-macrolide combination treatment in moderately severe community-acquired pneumonia: a randomized noninferiority trial JAMA Intern Med 2014; 174: 1894-1901 
65 Postma DF, van Werkhoven $C H$, van Elden $L J$ et al. Antibiotic treatment strategies for community-acquired pneumonia in adults. N Engl J Med 2015; 372: 1312 - 1323

66 Araujo FG, Slifer TL, Remington JS. Effect of moxifloxacin on secretion of cytokines by human monocytes stimulated with lipopolysaccharide. Clin Microbiol Infect 2002; 8: 26-30

67 Dalhoff A. Immunomodulatory activities of fluoroquinolones. Infection 2005; 33 : (Suppl. 02): $55-70$

68 Donnarumma G, Paoletti I, Buommino E et al. Anti-inflammatory effects of moxifloxacin and human beta-defensin 2 association in human lung epithelial cell line (A549) stimulated with lipopolysaccharide. Peptides 2007; 28: 2286-2292

69 Weiss T, Shalit I, Blau H et al. Anti-inflammatory effects of moxifloxacin on activated human monocytic cells: inhibition of NF-kappaB and mitogen-activated protein kinase activation and of synthesis of proinflammatory cytokines. Antimicrob Agents Chemother 2004; 48: $1974-1982$

70 Muller-Redetzky HC, Wienhold SM, Berg J et al. Moxifloxacin is not antiinflammatory in experimental pneumococcal pneumonia. J Antimicrob Chemother 2015; 70: 830-840

71 Brunkhorst FM, Oppert M, Marx G et al. Effect of empirical treatment with moxifloxacin and meropenem vs meropenem on sepsis-related organ dysfunction in patients with severe sepsis: a randomized trial. JAMA 2012; 307: 2390-2399

72 Czyzyk TA, Ning Y, Hsu MS et al. Deletion of peptide amidation enzymatic activity leads to edema and embryonic lethality in the mouse. Dev Biol 2005; 287: 301 - 313

73 Caron KM, Smithies O. Extreme hydrops fetalis and cardiovascular abnormalities in mice lacking a functional Adrenomedullin gene. Proc Natl Acad Sci U S A 2001; 98: 615-619

74 Ichikawa-Shindo Y, Sakurai T, Kamiyoshi A et al. The GPCR modulator protein RAMP2 is essential for angiogenesis and vascular integrity. J Clin Invest 2008; 118: 29-39

75 Dackor RT, Fritz-Six K, Dunworth WP et al. Hydrops fetalis, cardiovascular defects, and embryonic lethality in mice lacking the calcitonin receptor-like receptor gene. Mol Cell Biol 2006; 26: 2511 - 2518

76 Agorreta J, Zulueta JJ, Montuenga LM et al. Adrenomedullin expression in a rat model of acute lung injury induced by hypoxia and LPS. Am J Physiol Lung Cell Mol Physiol 2005; 288: L536-545

77 Cheung BM, Hwang IS, Li CY et al. Increased adrenomedullin expression in lungs in endotoxaemia. J Endocrinol 2004; 181: $339-345$

78 Matheson PJ, Mays MP, Hurt RT et al. Adrenomedullin is increased in the portal circulation during chronic sepsis in rats. Am J Surg 2003; 186: $519-525$

79 Dackor $R$, Caron $K$. Mice heterozygous for adrenomedullin exhibit a more extreme inflammatory response to endotoxin-induced septic shock. Peptides 2007; 28: $2164-2170$

80 Hippenstiel S, Witzenrath $M$, Schmeck B et al. Adrenomedullin reduces endothelial hyperpermeability. Circ Res 2002; 91: 618-625

81 Itoh T, Obata H, Murakami S et al. Adrenomedullin ameliorates lipopolysaccharide-induced acute lung injury in rats. Am J Physiol Lung Cell Mol Physiol 2007; 293: L446 - 452

82 Temmesfeld-Wollbruck B, Brell B, David I et al. Adrenomedullin reduces vascular hyperpermeability and improves survival in rat septic shock. Intensive Care Med 2007; 33: 703 - 710

83 Müller HC, Witzenrath M, Tschernig T et al. Adrenomedullin attenuates ventilator-induced lung injury in mice. Thorax 2010; 65: 1077-1084

84 Muller-Redetzky HC, Will D, Hellwig $K$ et al. Mechanical ventilation drives pneumococcal pneumonia into lung injury and sepsis in mice: protection by adrenomedullin. Crit Care 2014; 18: R73

85 David S, Kumpers $P$, van Slyke P et al. Mending leaky blood vessels: the angiopoietin-Tie2 pathway in sepsis. J Pharmacol Exp Ther 2013; 345: 2-6

86 Eklund L, Saharinen P. Angiopoietin signaling in the vasculature. Exp Cell Res 2013; 319: 1271 - 1280

87 Eklund L, Olsen BR. Tie receptors and their angiopoietin ligands are context-dependent regulators of vascular remodeling. Exp Cell Res 2006; 312: 630-641

88 Scharpfenecker $M$, Fiedler $U$, Reiss $Y$ et al. The Tie-2 ligand angiopoietin2 destabilizes quiescent endothelium through an internal autocrine loop mechanism. J Cell Sci 2005; 118: 771 - 780

89 Fiedler $U$, Reiss $Y$, Scharpfenecker $M$ et al. Angiopoietin-2 sensitizes endothelial cells to TNF-alpha and has a crucial role in the induction of inflammation. Nat Med 2006; 12: 235 -239
90 Parikh SM, Mammoto T, Schultz A et al. Excess circulating angiopoietin-2 may contribute to pulmonary vascular leak in sepsis in humans. PLoS Med 2006; 3: e46

91 Calfee CS, Gallagher D, Abbott J et al. Plasma angiopoietin-2 in clinical acute lung injury: prognostic and pathogenetic significance. Crit Care Med 2012; 40: $1731-1737$

92 David S, Mukherjee A, Ghosh CC et al. Angiopoietin-2 may contribute to multiple organ dysfunction and death in sepsis. Crit Care Med 2012; 40: 3034 - 3041

93 Ziegler T, Horstkotte J, Schwab C et al. Angiopoietin 2 mediates microvascular and hemodynamic alterations in sepsis. J Clin Invest 2013; 123: $3436-3445$

94 Mammoto T, Parikh SM, Mammoto A et al. Angiopoietin-1 requires p190 RhoGAP to protect against vascular leakage in vivo. J Biol Chem 2007; 282: 23910-23918

95 Witzenbichler B, Westermann D, Knueppel S et al. Protective role of angiopoietin-1 in endotoxic shock. Circulation 2005; 111: 97 - 105

96 McCarter SD, Mei SH, Lai PF et al. Cell-based angiopoietin-1 gene therapy for acute lung injury. Am J Respir Crit Care Med 2007; 175: 1014- 1026

$97 \mathrm{Xu} \mathrm{J,} \mathrm{Qu} \mathrm{J,} \mathrm{Cao} \mathrm{L} \mathrm{et} \mathrm{al.} \mathrm{Mesenchymal} \mathrm{stem} \mathrm{cell-based} \mathrm{angiopoietin-1}$ gene therapy for acute lung injury induced by lipopolysaccharide in mice. J Pathol 2008; 214: 472-481

98 David S, Park JK, Meurs M et al. Acute administration of recombinant Angiopoietin-1 ameliorates multiple-organ dysfunction syndrome and improves survival in murine sepsis. Cytokine 2011; 55: 251 - 259

99 Huang YQ Sauthoff H, Herscovici Pet al. Angiopoietin-1 increases survival and reduces the development of lung edema induced by endotoxin administration in a murine model of acute lung injury. Crit Care Med 2008; 36: $262-267$

$100 \mathrm{Mei}$ SH, McCarter SD, Deng Y et al. Prevention of LPS-induced acute lung injury in mice by mesenchymal stem cells overexpressing angiopoietin 1. PLoS Med 2007; 4: e269

101 Tournaire $R$, Simon MP, le Noble F et al. A short synthetic peptide inhibits signal transduction, migration and angiogenesis mediated by Tie2 receptor. EMBO Rep 2004; 5: 262 - 267

102 Kumpers P, Gueler F, David $S$ et al. The synthetic tie2 agonist peptide vasculotide protects against vascular leakage and reduces mortality in murine abdominal sepsis. Crit Care 2011; 15: R261

103 David S, Ghosh CC, Kumpers P et al. Effects of a synthetic PEG-ylated Tie-2 agonist peptide on endotoxemic lung injury and mortality. Am J Physiol Lung Cell Mol Physiol 2011; 300: L851 - 862

104 Sugiyama MG, Armstrong SM, Wang C et al. The Tie2-agonist Vasculotide rescues mice from influenza virus infection. Sci Rep 2015; 5: 11030

105 Cribbs SK, Matthay MA, Martin GS. Stem cells in sepsis and acute lung injury. Crit Care Med 2010; 38: 2379-2385

106 Mao M, Wang SN, Lv XJ et al. Intravenous delivery of bone marrow-derived endothelial progenitor cells improves survival and attenuates lipopolysaccharide-induced lung injury in rats. Shock 2010; 34 : $196-204$

107 Bhattacharya J, Matthay MA. Regulation and repair of the alveolar-capillary barrier in acute lung injury. Annu Rev Physiol 2013; 75: 593 615

108 Islam MN, Das SR, Emin MT et al. Mitochondrial transfer from bonemarrow-derived stromal cells to pulmonary alveoli protects against acute lung injury. Nat Med 2012; 18: 759 - 765

109 Burnham EL, Taylor WR, Quyyumi AA et al. Increased circulating endothelial progenitor cells are associated with survival in acute lung injury. Am J Respir Crit Care Med 2005; 172: 854-860

110 Luo TH, Wang Y, Lu ZM et al. The change and effect of endothelial progenitor cells in pig with multiple organ dysfunction syndromes. Crit Care 2009; 13: R118

111 Patschan SA, Patschan D, Temme J et al. Endothelial progenitor cells (EPC) in sepsis with acute renal dysfunction (ARD). Crit Care 2011; 15: R94

112 Lam CF, Liu YC, Hsu JK et al. Autologous transplantation of endothelial progenitor cells attenuates acute lung injury in rabbits. Anesthesiology 2008; 108: $392-401$

113 Fan H, Goodwin AJ, Chang E et al. Endothelial progenitor cells and a SDF-1alpha analogue synergistically improve survival in sepsis. Am J Respir Crit Care Med 2014; 189: 1509-1519

114 Muller-Redetzky H, Lienau J, Suttorp $N$ et al. Therapeutic strategies in pneumonia: going beyond antibiotics. Eur Respir Rev 2015; 24: 516 524 